\title{
Pseudoaneurisma gigante crónico compresivo de arteria ilíaca externa izquierda secundario a herida de arma de fuego
}

\author{
Compressive chronic giant pseudoanerysm of the left external iliac \\ artery secondary to gunshot wound
}

Federico Martín Riolo, Patricio Rattagan, Jorge Orlando Cortez Yáñez, Miguel Osvaldo Villegas

\begin{abstract}
RESUMEN
Los pseudoaneurismas ilíacos postraumáticos son poco frecuentes. Presentamos un paciente masculino de 44 años con antecedentes herida de arma de fuego y requerimiento de colostomía con reconstrucción del tránsito. Consulta 13 años después por masa pulsátil dolorosa en abdomen asociada a dolor en reposo del miembro inferior izquierdo (Fontaine III - Rutherford IV) de dos años de evolución. La angio-TC evidenció pseudoaneurisma de arteria ilíaca externa izquierda de morfología sacular y diámetro máximo de $213 \mathrm{~mm}$. El abordaje endovascular con tres stents graft fue exitoso. La angio-TC demostró buen resultado. Presentamos el enfoque diagnóstico, terapéutico y seguimiento al año.
\end{abstract}

Palabras clave: pseudoaneurisma, pseudoaneurisma postraumático, falso aneurisma, herida de arma de fuego, angioplastia iliaca.

\begin{abstract}
Posttraumatic iliac pseudoaneurysms are rare. We present a 44 year old male patien with a history of gun injury and colostomy requirement with reconstruction of the traffic. Consultation 13 years later for painful pulsatile mass in the abdomen associated with resting pain of the lower left limb (Fontaine III - Routherford IV) two years later. Angio-CT showed pseudoaneurysm of the left external iliac artery of sacular morphology and a maximum diameter of $213 \mathrm{~mm}$. The endovascular approach with three graft stents was successful. Angio-CT showed good result. We present the diagnostic, therapeutic and one year follow up approach.
\end{abstract}

Keywords: pseudoaneurysm, postraumatic pseudoaneurysm, false aneurysm, gunshot wound, iliac angioplasty.

Revista Argentina de Cardioangiología Intervencionista 2020;11(1):35-37. https://doi.org/10.30567/RACI/202001/0035-0037

\section{INTRODUCCIÓN}

Los pseudoaneurismas ilíacos postraumáticos son poco frecuentes. La rareza de su aparición luego de heridas por arma de fuego (HAF) es evidenciado por los pocos casos descritos en la literatura.

Los pseudoaneurismas surgen de una interrupción en la continuidad de la pared arterial como resultado de la inflamación, trauma o causas iatrogénicas. Bajo la influencia de la presión arterial sostenida, la sangre diseca en los tejidos alrededor de la arteria y forma un saco perfundido que se comunica con la luz arterial. El saco perfundido está contenido por la túnica media o adventicia o simplemente por estructuras de tejidos blandos que rodean el vaso lesionado.

Presentamos el caso de un paciente de 44 años con antecedentes de colostomía por HAF y reconstrucción del tránsito. Evoluciona con pseudoaneurisma en la AIEI que se comporta como compresivo en las estructuras adyacentes, consultando 13 años posteriores al hecho. El paciente se sometió a tratamiento endovascular. Presentamos el enfoque diagnóstico, terapéutico y seguimiento al año.

\section{OBJETIVO}

Presentar un caso de pseudoaneurisma postraumático gigante, con evolución crónica de arteria ilíaca externa izquierda

1. Servicio de Hemodinamia. Hospital Nacional "Profesor Alejandro Posadas", El Palomar, Provincia de Buenos Aires, Rep. Argentina.

$\triangle$ Correspondencia: Miguel Osvaldo Villegas. Moreno 827, B2804FTO Campana, Bs. As. Rep. Argentina.Tel.:011-4469-2300 int.: 1105.miguelovillegas@gmail.com

Los autores no declaran conflictos de intereses

Recibido: 11/12/2019|Aceptado: 31/01/2020
(AIEI) y oclusión de esta, tratado con stents forrados (SF) por vía retrógrada, desde un acceso no convencional y seguimiento ambulatorio.

\section{CASO CLÍNICO}

Paciente masculino de 44 años. Antecedentes de HAF con requerimiento de colostomía intestinal y reconstrucción del tránsito. Consulta13 años después por masa pulsátil dolorosa a la palpación en abdomen y dolor en reposo del miembro inferior izquierdo (Fontaine III - Routherfor IV) desde hace 2 años. La angio-TC (Figura 1) evidenció la presencia de pseudoaneurisma sacular en la AIEI (171x165×213 mm), con desplazamiento de estructuras adyacentes a nivel pélvico y abdominal. Se observó adelgazamiento y oclusión de la luz de la arteria ilíaca externa por compresión extrínseca y sitio de entrada del pseudoaneurisma.

Por método de Seldinger se colocó vaina introductora $5 \mathrm{Fr}$ en arteria femoral común derecha. Se avanzó catéter $\mathrm{S} 1$ posicionándolo a nivel de la arteria ilíaca primitiva izquierda. En la angiografía control se visualizó oclusión y adelgazamiento de la AIEI. Se decidió realizar terapéutica por vía retrógrada con controles angiográficos por vía anterógrada, por lo cual se colocó un introductor 7 Fr. en la arteria femoral profunda izquierda. Se administraron $60 \mathrm{UI} / \mathrm{kg}$ de heparina. Por vaina 7 Fr se progresó alambre guía 0.035 " hidrofílico (Radifocus Terumo,Tokio, Japón) apoyado sobre catéter tipo Cobra hacia la aorta abdominal. Una vez franqueada la lesión se dilató con catéter balón 5x120 mm (Mustang, Boston, Massachusetts, USA) a nivel de la AIEI, sin complicaciones. Posteriormente, y en forma telescopada, se implantaron tres SF balón expandibles 8x59 mm (Advanta V12, Maquet, Rastatt, Alemania). Finalmente se realizó control angiográfico donde se observó la AIEI permeable con sellado y ex- 


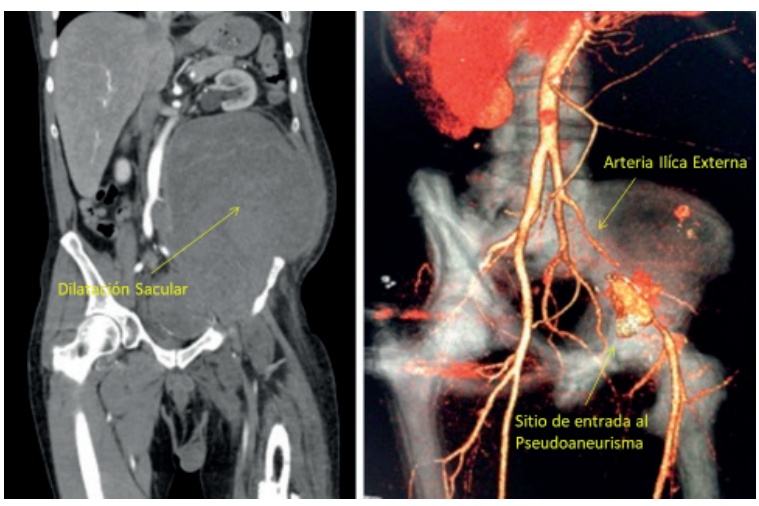

Figura 1. Angio-TC. A. Dilatación sacular $(171 \times 165 \times 213 \mathrm{~mm})$ en pelvis desplazando estructuras adyacentes a nivel abdominopélvico. B. Reconstrucción vascular: se observa el adelgazamiento de la luz de la arteria ilíaca por la compresión ejercida por el pseudoaneurisma y el área amplia que realza con contraste correspondiente al sitio de entrada del pseudoaneurisma.

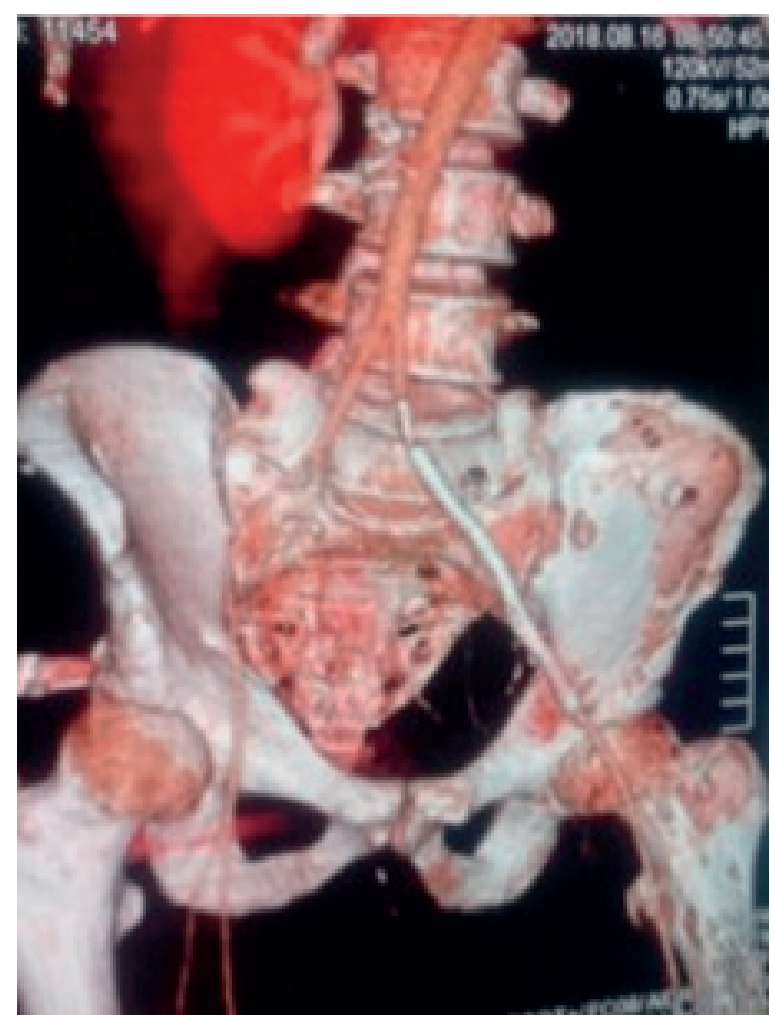

Figura 3. Angio-TC a los seis meses. Se observa la permeabilidad del eje iliofemoral izquierdo y la exclusión del pseudoaneurisma.

clusión completa del pseudoaneurisma (Figura 2). Evolucionó sin complicaciones, alta a las $24 \mathrm{hs}$. con indicación de doble antiagregación. Evolucionó a los 6 meses y al año asintomático y con ausencia de masa pulsátil. La angio-TC a los seis meses, AIEI permeable y disminución de $6 \mathrm{~cm}$ del tamaño del saco (Figura 3).

\section{DISCUSIÓN}

Los pseudoaneurismas de la AIE son causados por traumatismos, tumores, infecciones, vasculitis, aterosclerosis o complicaciones iatrogénicas ${ }^{1}$.

La mayoría de los pseudoaneurismas se descubren en el momento del traumatismo, pero existen casos de laceraciones arteriales que crecen progresivamente y originan un pseudoaneurisma años después ${ }^{2}$.

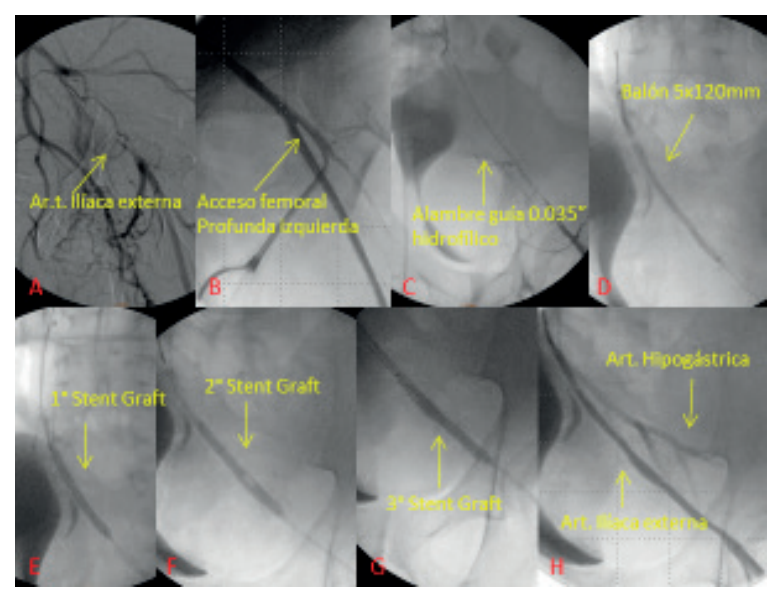

Figura 2. A. Acceso arterial femoral común derecho con introductor 5 Fr. Angiografía con catéter Simmons 1. Ilíaca externa izquierda de fino calibre colapsada. B. Acceso arterial femoral profunda izquierda (origen a nivel de la cabeza del fémur). C. Se avanza alambre guía hidrofilico apoyado sobre catéter tipo Cobra hasta llegar al nivel de la aorta abdominal-D. Predilatación con catéter balón $(5 \times 120 \mathrm{~mm})$. E, F, G. Se implantan tres stents graft de $8 \times 59 \mathrm{~mm}$ en forma telescopada. H. Angiografía final. Arteria ilíaca externa permeable y exclusión del pseudoaneurisma.

Los pseudoaneurismas pueden presentar complicaciones tales como tromboembolia, ruptura, compresión neurovascular, infección, anemia y muerte.

La tromboembolia y la anemia son las complicaciones más frecuentes, la ruptura puede ocurrir espontáneamente o durante un intento de oclusión por maniobra de compresión, principalmente en los no compresibles o solo compresibles con mucha fuerza. De tal forma, complicaciones importantes y graves como las arriba citadas pueden y deben ser evitadas, y la mejor manera de hacerlo es tratarlo a tiempo.

La forma de presentación más habitual a nivel ilíaco es el hallazgo incidental sin asociarse a ninguna manifestación clínica, pero pueden aparecer signos y síntomas derivados de la compresión local hasta en el $43 \%$ de los casos ${ }^{3}$. El dolor neuropático afecta al 9,5\% de los pacientes de la serie de Krups$\mathrm{ki}^{3}$ y hasta en un $20 \%$ de los pacientes de la serie de Gardiner ${ }^{4}$. Otros signos que se pueden asociar son dolor abdominal inespecífico (19\%), infección urinaria y hematuria (10\%), edema por compresión venosa (5\%), claudicación intermitente $(10 \%)^{3}$. La claudicación y la masa pulsátil fueron los síntomas y signos que manifestó nuestro paciente. Estos pseudoaneurismas generalmente permanecen en silencio; sin embargo, las consecuencias pueden ser mortales cuando se rompen. El diagnóstico de aneurismas y pseudoaneurismas ilíacos aislados puede confirmarse mediante ecografía, tomografía computarizada, resonancia magnética y arteriografía. La ecografía es mucho menos costosa que las otras modalidades, depende del operador y a menudo es imprecisa debido a la profundidad de las arterias en la pelvis o la presencia de gas intestinal suprayacente.

En relación con el tratamiento podemos citar seis modalidades: expectante, quirúrgico, compresión a ciegas o guiada por ultrasonido, inyección salina guiada por ultrasonido o angiografía seguida de compresión endovascular utilizando SF o coils, inyección de trombina guiada por angiografía o por ultrasonido. Diversos trabajos en la bibliografía citan que los pequeños con diámetros $<2 \mathrm{~cm}$ tienden a cerrarse a través de trombosis espontánea. La cirugía se reserva cuando existe ruptura, compresión de estructuras neurovasculares adyacentes, isquemia de la piel, dolor intenso y que presenten rápido crecimiento. Son contra- 
indicaciones al tratamiento por compresión los no compresibles o compresibles con oclusión arterial o acentuada reducción del flujo distal, localización arriba del ligamento inguinal, presencia de infección, trombosis venosa, isquemia del miembro o de la piel, compresión neurovascular, síndrome compartimental'5. La terapia endovascular tiene una morbimortalidad menor que la cirugía convencional con buenos resultados a mediano plazo, por lo que es una alternativa que hay que tener en cuenta en pacientes con elevado riesgo quirúrgico o antecedentes de cirugía abdominal previa ${ }^{6}$. Utilizar un SF para la exclusión de pseudoaneurismas se realiza actualmente para reemplazar la reparación quirúrgica abierta ${ }^{7}$.

Si el cuello del pseudoaneurisma es ancho es preferible utilizar SF o la opción quirúrgica, debido al elevado riesgo de embolización de los coils o el material embolizante utilizado. Sin embargo, deben evitarse los SF en vasos terminales o de fino calibre por su elevada tasa de trombosis y reestenosis ${ }^{8}$. En nuestro caso, la decisión de optar por tratamiento endovascular se basó en el antecedente de cirugía abdomino-pélvica en con- texto de herida de arma de fuego lo cual dificultaría un segundo acceso asociado a la necesidad de revascularización de la AIEI debido a su compresión por el gran volumen del pseudoaneurisma. La utilización de trombina y coils no sería la recomendable debido al gran volumen y al cuello amplio. La compresión no sería de utilidad por encontrarse arriba del conducto inguinal.

El empleo de los SF o endoprótesis se ha aplicado con éxito en la exclusión de pseudoaneurismas desde la década de los noventa.

\section{CONCLUSIÓN}

Las complicaciones secundarias al trauma son en gran parte consecuencia de retrasos o desaciertos ya sean del diagnóstico, tratamiento o seguimiento y pueden presentarse a corto, mediano o largo plazo. El tratamiento endovascular es una alternativa en pacientes con elevado riesgo quirúrgico o antecedentes de cirugía abdominal previa. El fin principal del tratamiento quirúrgico en lesiones vasculares debe ser el control de la hemorragia.

\section{BIBLIOGRAFÍA}

1. SueyoshiE, Sakamotol, Nakashima K, MinamiK, HayashiK. Visceral andperipheral arterial pseudoaneurysms. AJR Am J Roentgenol 2005;185:741-9.

2. Papadakos N, Wales L, Hayes K, Belli AM, Loftus I, Ray S. Post-traumatic pelvic pseudoaneurysm and arterio-venous fistula: combined endovascular and surgical approach. Eur J Vasc Endovasc Surg 2008:36:164-6.

3. KrupskiW, Selzman C, Floridia R, StreckerP, Nehler M, Whitehill T. Contemporarymanagement of isolatediliac aneurysms.J Vasc Surg 1998;28:1-13.

4. Gardiner M, Mangwani J, Williams WW. Aneurysm of the common iliac artery presenting as a lumbosacral plexopathy. I Bone Joint Surg Br 2006;88:1524-6.

5. dos Santos Nogueira AC, Gonzalez Salgado C, Belloni dos Santos Nogueira F, Israel do Amaral S, Rabischoffsky A. Hospital Pro-Cardía-

Co, Rio de Janeiro, RJ - Brasil Ara Bras Cardiol: imagem cardiovasc 2013;26(4):289-307.

6. Gallego-Ferreiro C, Vidal-Rey J, Encisa de Sá JM, Rosendo-Carrera A. Tratamiento endovascular de un pseudoaneurisma ilíaco postraumático: a propósito de un caso. Angiología 2009;61(3):147-52.

7. Ricciardi E, Di Martino G, Maniglio P, et al. Life-threatening bleeding after pelvic lymphadenectomy for cervical cancer: endovascular management of ruptured false aneurysm of the external iliac artery. World I Surg Oncol 2012;10:149.

8. Girassolli A, Rattagan P, Garré L, et al. Manejo endovascular de un pseudoaneurisma gigante de la arteria femoral superficial. Revista Argentina de Cardioangiología Intervencionista 2017;(2):0086-0088 